\title{
A Study of Professional Deformation and Psychological Defense of a Practitioner Psychologist's Personality at Various Stages of Professional Activity
}

\section{Svetlana Kuzenko}

\author{
Candidate of Psychological Sciences, Associate Professor of the Chair of Psychophysiology and Clinical Psychology of the \\ Academy of Psychology and Pedagogy at Southern Federal University; Sania777_83@mail.ru
}

\section{Doi:10.5901/mjss.2015.v6n6s5p169}

\section{Abstract}

\begin{abstract}
The goal of the empirical part of this work is to study the use of psychological defenses by practitioner psychologists and development of job burnout in them. Giessen Psychosomatic Complaint Questionnaire, Cattell's Test, 16 Personality Factors Questionnaire (16 PFQ-187-A) by R.B. Cattell, S. Rosenzweig Picture Frustration Study, and V.V. Boiko's technique of diagnosing the dominant strategy of psychological defense in communication are used in the study. For our study, we have selected a battery of diagnostic techniques helping to identify the mechanisms used by practitioner psychologists; the extent of professional deformation of psychologists was studied taking into account the duration of professional activity. The interrelation between psychological defense mechanisms used by practitioner psychologists and the level of their professional deformation was detected. Using these diagnostic techniques, we studied the personality traits of practitioner psychologists at different stages of their activity and discovered the range of practicing psychologist's work experience located in the "risk zone" of the professional deformation development.
\end{abstract}

Keywords: psychological defense, frustration, professional deformation, deflexion, projection.

\section{Introduction}

The study of professional deformation of practitioner psychologists in connection with the growing demand to the representatives of this profession and the application of their knowledge and skills in many spheres of life are of great importance.

The specialists' using the psychological defense influences the quality of the psychological work and can contribute to a successful activity or to formation of professional deformation.

Deformation (from Latin deformation - distortion) refers to the deviation from the initially correct ideal, plan or model caused by the influence of external factors on the object. These features can be incorporated more deeply into the structure of personality, rendering it into hierarchy, which reminds the change occurring in an individual in case of accentuations. The studies have shown that there is no fatal pre-determinacy of professional deformation for each worker. There is a certain permissible limit of professional deformation (Makarova; Greenberger, 1981).

The professional deformation includes two components: initial inclination and the professional deformation itself. Initial inclinations refer to those traits of character and personal features, which are manifested in the representatives of certain profession before their contact with future job. They may be understood by their owners, be accepted as desirable and invoke a sense of pride. Or, on the contrary, be unconscious, suppressed and hidden.

The process of "professional deformation" is not irreversible, if pay attention to it in proper time. There are special methods of work with professional deformation and psycho-emotional burnout. In particular, here the trainings on personal and professional growth, reflexion of professional development and drafting of alternative scenarios of professional life, as well as individual counseling, forecast of the desired professional achievements and other forms of work may be useful. These measures may be efficient enough, not only when eliminating the negative consequences, but also when preventing the unwanted professional development of practicing psychologist (Maslach, 1978).

\section{Goals and Objectives of the Research}

The goal of the empirical part of this work is to study the use of psychological defenses by practitioner psychologists and development of job burnout in them. Attaining the goal involves meeting the following objectives:

1. Using the above diagnostic techniques, to study the personal traits of practitioner psychologists in various stages of activity; 
2. To study the extent of professional deformation of psychologists, taking into account the duration of professional activity.

3. To identify the relationship between the mechanisms of psychological defenses used by practitioner psychologists and their professional deformation level.

The experimental bases of our study are the practitioner psychologists who work in gestalt-approach psychotherapy direction. The total number of research subjects amounted to 156 people, aged 21-55 years, with the average age of 31.5 years.

\section{Methods, Used in the Study}

For this study the following techniques have been selected:

- Giessen Psychosomatic Complaint Questionnaire.

Giessen Psychosomatic Complaint Questionnaire (Giesener Beschwerdebogen, GBB) is a questionnaire designed to register the individual complaints, series of complaints and to give the overall estimation of their intensity.

GBB is not a questionnaire of somatic diseases symptoms, it includes only somatic symptoms, occurring in the picture of psychosomatic disorders.

- Cattell's test. Sixteen Personality Factor Questionnaire (16 Personality Factors) by R.B. Cattell. 16 PFQ-187-A

16-Personality Factor Questionnaire by R.B. Cattell (Form A) can reveal the features of character, inclinations and interests of the individual. Cattell's Questionnaire is one of the most well-known multi-factorial techniques, created in the context of objective experimental approach to the study of personality. According to the Cattell's theory of personality traits, the personality is described as consisting of stable, steady, interconnected elements (properties, traits) that define its inner nature and behavior. Differences in human behavior are explained by the differences in intensity of personality traits.

- S. Rosenzweig Picture Frustration Technique.

This technique is used for studying the reactions to failure and ways to manage situations that prevent activities or satisfaction of the needs of the individual.

- V.V. Boiko's techniques for diagnosing the dominant strategy of psychological defense in communication (Boiko, 1996; Vodopianova \& Starchenkova, 2009).

This method is used for diagnostics of the leading defense strategy in communication with partners.

It includes 3 scales: Peacefulness, Avoidance, Aggression.

The more answers belong to one type, the more distinctly expressed this strategy is; if the quantity of answers is approximately equal, it means that in contact with partners the testee actively uses various types of defense of his subjective reality (Vodopianova \& Starchenkova, 2009).

\section{Research Findings Processing and Analysis Methods}

In this work we used the following research methods:

- empirical methods: questionnaire, survey, quantitative and qualitative data analysis;

- statistical methods: descriptive statistics, Kruskal-Wallis H-criterion, Pearson correlation coefficient. Microsoft Excel and Statistica 17.0 software were used for mathematical data processing.

Reliability and objectivity of the results is ensured by the methodological feasibility of empirical research procedures, using the methods of mathematical-statistical data processing.

In context of statistical processing of the results using the Kolmogorov-Smirnov $\lambda$ - criterion we verified the distribution of the received data. In most variables the distribution differed from a normal, therefore, we later used nonparametric methods of static analysis: Pearson correlation coefficient and nonparametric Kruskal-Wallis $\mathrm{H}$-criterion.

\section{Results and Discussion}

5.1 Study of relations of the used psychological defenses and extent of somatic complaints in the research subjects from three groups, divided by the duration of psychological practice duration.

The testees were divided into three groups in accordance with the duration of professional activity. The first group included people with work experience from 2 months to 2 years, aged 21-33, in the amount of 54 people; the second 
group - from 2 to 5 years of practice, aged 23-39, in the amount of 42 people; the third group - more than 5 years of psychological practice, aged $32-55$, in the amount of 60 people.

The research subjects were tested individually. The testees received instructions and questionnaire forms. After the test all the data has been processed and summarized in raw indicators tables.

Further the extent of indicators of the study results in percentage ratio was calculated, the statistical analysis of groups by Kruskal-Wallis $\mathrm{H}$-criterion $\mathrm{H}$-criterion was conducted and the Pearson correlation coefficients were calculated according to the extent of interrelationship of psychological defenses and extent of somatic complaints. Based on this data we have attempted to trace the impact of psychological defenses of respondents on the extent of somatic complaints extent (as one of the factors of manifestations of job burnout in psychologists) at different stages of the professional activity. We have investigated the relationship between the psychological defenses used by psychologists and the development of professional deformation.

In our study of psychological defenses we used the "Methods of diagnostics of the dominant strategy of psychological defense in communication by V.V. Boiko" and compared the scales of these methods with the theoretical basis of psychological defenses in Gestalt-therapy. In the questionnaire by V.V. Boiko there are 3 scales: "Peacefulness", "Avoidance", "Aggression". The scale of "Peacefulness" we compared with psychological defenses "Introjection / Retroflexion" the scale "Avoidance" - with the defense "Deflexion", and the scale "Aggression" - with "Projection". In Gestalt-therapy these defenses are called the means of interrupting contact which include Confluence, Projection, Retroflexion, Introjection (Boiko,1996).

As a result of data processing we have identified in the first group of research subjects that $27.9 \%$ of the testees have a high score of "Pressure of somatic complaints" where the components of this scale are: $72,2 \%$ of the testees have high scores of "Exhaustion", "44,4\% of the testees have high scores of "Rheumatic complaints", 5,6\% of the testees have "Gastric complaints"'" , 5,6\% of the testees have "Heart complaints". We can see that high scores on "Pressure of somatic complaints" in the research subjects of the first group is formed as a result of increasing of "Exhaustion" and "Rheumatic complaints", and we can assume that these factors promote formation of somatic complaints and, as a consequence, the professional deformation at the initial stages of psychological practice.

Table 1. Percentage ratio of the extent of somatic complaints in practitioner psychologists at the stage of professional activity lasting from 2 months to 2 years

\begin{tabular}{|c|c|c|c|c|c|c|}
\hline $\begin{array}{c}\text { Groups according to the } \\
\text { work experience }\end{array}$ & $\begin{array}{c}\text { Extent of somatic } \\
\text { complaints }\end{array}$ & "Exhaustion" & $\begin{array}{c}\text { "Rheumatic } \\
\text { complaints" }\end{array}$ & $\begin{array}{c}\text { "Gastric } \\
\text { complaints" }\end{array}$ & $\begin{array}{c}\text { "Heart } \\
\text { complaints" }\end{array}$ & $\begin{array}{c}\text { Pressure of complaints } \\
\text { (overall scores) }\end{array}$ \\
\hline $\begin{array}{c}\text { 1st group } \\
\text { (work experience from } \\
\text { 2months to 2 years) }\end{array}$ & Low level & $27.8 \%$ & $55.6 \%$ & $94.4 \%$ & $94.4 \%$ & $72.2 \%$ \\
\cline { 2 - 7 } & High level & $72.2 \%$ & $44.4 \%$ & $5.6 \%$ & $5.6 \%$ & $\underline{\mathbf{2 7 . 8} \%}$ \\
\hline
\end{tabular}

The psychologists of the second group prefer to use the defense "Introjection / Retroflexion" - 63\% of the testees, $21.3 \%$ of the testees prefer to use the defense "Deflexion" and $15.7 \%$ of the testees use the defense "Projection". In most cases, the newcomer psychologists let in themselves the certain ideas, attitudes, beliefs, concepts, relations, standards of behavior, moral, values, etc. from other people without checking this material critically.

Table 2. Percentage ratio of the use of psychological defenses by practitioner psychologists at the stage of professional activity lasting from 2 months to 2 years

\begin{tabular}{|l|c|}
\hline Psychological defenses & 1st group \\
\hline "Introjection / Retroflexion" (Peacefulness) & $63 \%$ \\
\hline "Deflexion" (Avoidance) & $21.3 \%$ \\
\hline "Projection" (Aggression) & $15.7 \%$ \\
\hline
\end{tabular}

The correlation analysis of psychological defense "Introjection / Retroflexion" (Peacefulness) and somatic complaints in the first group the following was revealed: moderate negative relationship $(r=-0.547)$ between the high statistical significance $(p=0.000)$ and somatic "Exhaustion"; moderate negative relationship $(r=-0.478)$ between high statistical significance $(p=0.000)$ and "Rheumatic complaints; moderate negative relationship $(r=-0.457)$ between the medium strength of statistical significance $(p=0.001)$ and "Heart complaints; moderate negative relationship $(r=0.557)$ between the high statistical significance $(p=0.000)$ and "Pressure of somatic complaints".

$63 \%$ of the testees in the first group more often use the psychological defense "Introjection / Retroflexion", and we 
can assume that it helps to reduce the somatic "Exhaustion", reducing the "Rheumatic complaints", "Heart complaints", reducing the overall "Pressure of somatic complaints".

The features of research subjects in the first group (27.9\%), who have high scores on somatic complaints: they are typical of personal features: sensitivity, dreaminess, dominance, suspicion, radicalism, straightforwardness, calmness. These psychological defenses are used to a larger extent: "Introjection / Retroflexion" (37\%) and "Projection" (37\%) and, to a smaller extent-"Deflexion" (26\%). The testees with low somatic complaints in the first group use to a larger extent the defense "Introjection / Retroflexion"-73.2\%, and to a smaller extent"Deflexion"-19.2\% and "Projection" - 7.6\%. We can assume that at the initial stages of psychological practice the use of defense "Projection" can promote increasing of somatic complaints and, as a consequence, of professional deformation.

In the second group of research subjects with psychological practice experience from 2 to 5 years we have identified that $50 \%$ of the respondents have the high scores of "Pressure of somatic complaints" where the components of this scale - $64.3 \%$ of the testees have high scores of "Exhaustion", "57.2\% of the testees have high scores of "Rheumatic complaints", $35.7 \%$ of the testees have "Gastric complaints", 14.3\% of the testees have "Heart complaints" . The percentage rate of "Pressure of somatic complaints" in this group has increased compared to the first group. We can also observe an increase in per cent on the scale of "Exhaustion", "Rheumatic complaints", "Gastric complaints".

The research subjects equally prefer to use the defenses "Introjection / Retroflexion"-45\% of the testees and "Deflexion"- is also 45\%, and only 10\% use the defense "Projection". We are seeing a trend, characteristic to the testees in the first group with high somatic complaints - equal ratio in the use of defenses "Introjection / Retroflexion" and "Deflexion".

The correlation analysis of psychological defense "Introjection / Retroflexion" in the second group revealed a moderate negative relationship of the low statistical significance with "Exhaustion" ( $r=-0.277 ; p=0.043)$, with "Rheumatic complaints $(r=-0,272 ; p=0,047)$ and moderate negative relationship of low statistical significance with "Pressure of somatic complaints" $(r=-0.307 ; p=0.024)$, and moderate negative relationship of high statistical significance with "Deflexion" $(r=-0.566 ; p=0.000)$ and the medium statistical significance with "Projection" $(r=-0.379 ; p$ $=0.005$ ).

The testees in the second group who have high somatic complaints use to a larger extent the psychological defense "Deflexion"- $56 \%$ of the testees, to a smaller extent"Introjection / Retroflexion"- $42 \%$ of the testees, "Projection"2\%. Their typical personal features are- dominance (42.9), courage (28.6), sensitivity (42.9), straightforwardness (28.6). The testees in the second group showing low somatic complaints to a larger extent use "Introjection / Retroflexion"- 45\%, to a smaller extent "Deflexion" - 38\%, and $17 \%$ use the defense "Projection". The trend to increasing of somatic complaints when applying defense "Deflexion" has been verified.

In the third group with work experience of psychological practice over 5 years we identified the high scores of "Pressure of somatic complaints" in $35 \%$ of the testees. It is $7.2 \%$ more than in the first group, but $15 \%$ less than in the second group. The components of the scale "Pressure of somatic complaints" - 70\% of the testees have high scores on the scale of "Exhaustion", 58.3\% of the testees have high scores of "Rheumatic complaints", $10 \%$ of the testees have high scores of "Gastric complaints", 30\% have high scores of "Heart complaints". The percentage rate of "Pressure of somatic complaints" in this group has increased compared to the first group.

The research subjects prefer to use the defenses "Introjection / Retroflexion" - $76 \%$ of the testees, to a smaller extent - "Deflexion"- 18\%, and only $6 \%$ use the defense "Projection". The testees in the second group, who have high somatic complaints,64\% use the psychological defense "Introjection / Retroflexion" and 36\% use "Deflexion". We are seeing a trend, characteristic to the testees in the first group with high somatic complaints - to some extent equal ratio in the use of defenses "Introjection / Retroflexion" and "Deflexion". The testees in the third group showing low somatic complaints to a larger extent use "Introjection / Retroflexion" - 83\%, to a smaller extent "Deflexion" - 8.5\%, and and "Projection" - 8.5\%. The trend to increasing of somatic complaints when applying defense "Deflexion" has been verified. (Table: Percentage ratio of the use of psychological defenses by practitioner psychologists at the stage of professional activity of over 5 years).

The analysis of psychological defense "Introjection / Retroflexion" in the third group revealed the moderate negative relationship of high statistical significance with "Deflexion" $(r=-0.566 ; p=0.000)$. That is, with increasing use of psychological defense "Introjection / Retroflexion" the use of "Deflexion" is reducing. No statistically significant correlations of "Introjection / Retroflexion" with somatic complaints in the third group have been identified.

Bearing in mind the above, we can assume that the extent of somatic complaints is inversely related to the psychological defense "Introjection / Retroflexion" and is directly related to the psychological defense "Deflexion" that is the use of defense mechanisms "Introjection / Retroflexion" reduces somatic complaints and prevents the professional deformation (Miroshnichenko, 2014). 


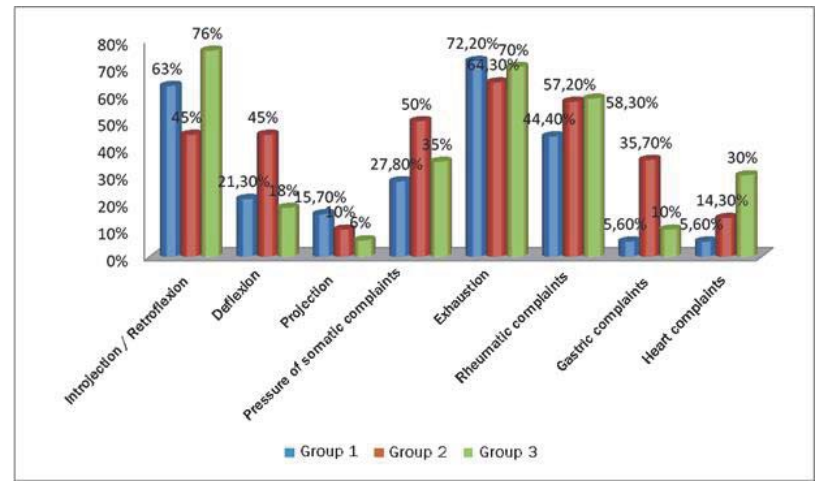

Diagram 1. Correlation of psychological defenses and somatic complaints of practitioner psychologists at various stages of professional activity

Statistical analysis of the three groups according to Kruskal-Wallis H-criterion has revealed the statistically significant differences between the groups for "Gastric complaints". (Table. Comparing groups according to Kruskal-Wallis Hcriterion on the scale of "Gastric complaints"). The most expressed in percentage terms are "Gastric complaints" in the second group compared to 1st 3rd groups. The second group respondents are the risk group in relation to the development of professional deformation. A high ratio of "Gastric complaints" in the second group may indicate that the use of defense "Deflexion" promotes forming of gastric complaints. Metaphorically speaking, the "closedness" to contacts with other people may lead to "intolerance" to the client's problems and own problems, and the development of gastric complaints.

Statistical analysis of the three groups according to Kruskal-Wallis H-criterion has revealed the statistically significant differences between the groups for "Heart complaints". (Table 3). Comparing groups according to KruskalWallis H-criterion on the scale of "Heart complaints") The highly expressed ratio of high "Heart complaints" in the participants of the third group with psychological practice experience of over 5 years (30\% of the testees). Participants in general more often use the psychological defense "Introjection / Retroflexion" - 76\%, 13\% more compared with the first group, which may indicate that that intensive use of defense "Introjection / Retroflexion"can lead to the formation namely of "Heart complaints".

Table 3. Comparing groups according to Kruskal-Wallis H-criterion on the scale of "Heart complaints").

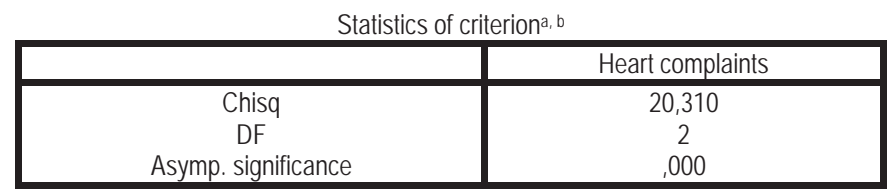

a Kruskal-Wallis H-criterion

b Grouping variable: groups

The statistically significant differences between the groups were revealed using Kruskal-Wallis $\mathrm{H}$-criterion on the psychological defense of "Introjection / Retroflexion". The lowest extent of the use of this defense in the second group is $45 \%$, where the highest ratio of somatic complaints (50\%). The third and first groups have the highest extent of the use of "Introjection / Retroflexion". We can assume that the psychologists that use this psychological defense, have a greater chance to have great work experience and reduce the level of professional deformation. 
Table 4. Comparing groups according to Kruskal-Wallis H-criterion on the scale "Introjection / Retroflexion"

Statistics of criterion ${ }^{a, b}$

\begin{tabular}{|c|c|}
\hline & Introjection / Retroflexion \\
\hline Chisq & 16,735 \\
DF & 2 \\
Asymp. significance &, 000 \\
\hline
\end{tabular}

a Kruskal-Wallis H-criterion

b Grouping variable: groups

Statistical analysis of the three groups according to Kruskal-Wallis H-criterion has revealed the statistically significant differences between the groups for the defense mechanism"Projection". Projection" is the most highly expressed in the first group of the three groups $-15.7 \%$. The third group has the lowest extent $-6 \%$. Since the heart complaints are expressed in the third group, the confirmed difference is statistically significant, we can assume that small percentage of use of projective mechanisms affects the severity of heart complaints. (Table 5. Comparing groups according to KruskalWallis H-criterion on the scale "Projection").

Table 5. Comparing groups according to Kruskal-Wallis H-criterion on the scale "Projection"

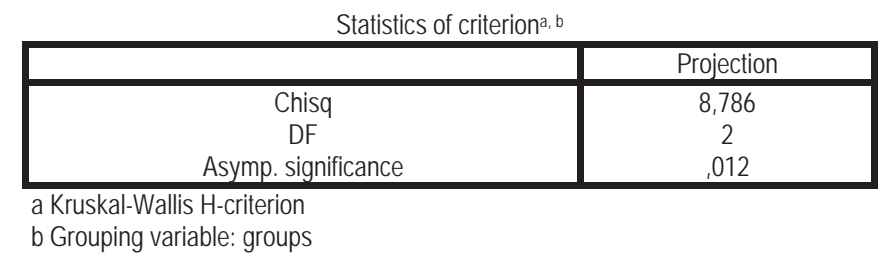

\subsection{Study of the interrelationship of psychological practice experience and personal features of practitioner} psychologists, behavioral strategies in frustration situations

The correlation analysis of Experience of psychological activities ranging from 0 to 2 years revealed: moderate negative relationship ( $r=-0.390)$ of medium strength statistical significance $(p=0.004)$ with psychological defense mechanism Introjection / Retroflexion (Peacefulness); moderate negative relationship ( $r=-0.420)$ of medium statistical significance $(p=0.002)$ with factor A-"Unsociability"; moderate negative relationship $(r=-0.369)$ of medium statistical significance $(p=0.006)$ with the factor F-"Restraint"; moderate negative relationship $(r=-0.355)$ of medium statistical significance $(p=0.008)$ with factor $\mathrm{H}$-"Shyness";"; moderate positive relationship $(r=0.370)$ of medium statistical significance $(p=0.006)$ with factor $L+\ll$ Suspicion".

Among the factors mentioned according to Cattell's test the factor $L+$ "Suspicion" is the most expressed in percentage calculation in the first group - in $39 \%$ of the testees.

We can assume that the research subjects in the initial stage of psychological practices have such qualities as wariness towards people, propensity to jealousy, desire to hold responsibility for errors on others, sourness.

These traits can promote reducing the use of psychological defense "Introjection / Retroflexion" and the possible increase in somatic complaints, and as a result, the development of professional deformation. We cannot affirm whether the formation of these traits is related to the work experience, , perhaps, these data is not related to their professional activities.

The correlation analysis of Experience of psychological activities ranging from 2 to 5 years revealed: moderate negative relationship $(r=-0.400)$ of medium statistical significance $(p=0.003)$ with factor E-"Obedience"; moderate negative relationship $(r=-0.456)$ of medium statistical significance $(p=0.001)$ with the factor F-"Restraint"; moderate negative relationship $(r=0.468)$ of high statistical significance $(p=0.000)$ with factor $\mathrm{H}$-"Shyness"; "Conformism". Increase of Impunitive directions of reactions in situations of frustration.

Psychologists with this level of experience are characterized by mildness and compliancy, try to follow certain rules, careful and prudent, sensible, prone to concerns, contained in emotions, they are shy, socially tactful. In situations of frustration they take the blame, and do not react to the environment.

The correlation analysis of Experience of psychological activities ranging from 2 to 5 years revealed no 
relations of high and medium statistical significance, and revealed a moderate relationship of low statistical significance with the factor $\mathrm{N}+$ "Diplomacy" and a weak negative relationship of low statistical significance with $\mathrm{E}$ in obstruction situation.

The Pearson correlation analysis was conducted without dividing participants into groups (see Table 6). The result is that with the increase of the length of psychological activity the "Heart complaints $(r=0.350 ; p=0.000)$ increase too, the use of psychological defense "Introjection / Retroflexion" ( $r=0.231 ; p=0.004)$ increases as well, and "Communicative" ( $r=0.247 ; p=0.002)$, "High intelligence" $(r=0.267 ; p=0.001)$,"Diplomacy" $(r=0.248 ; p=$ 0.002),"Conformism" ( $r=-0.227 ; p=0.004)$ occur.

Table 6. Results of comparative correlation analysis of work experience in all groups of psychologists

\begin{tabular}{|c|c|c|c|c|c|c|c|c|}
\hline & & $\begin{array}{c}\text { Heart } \\
\text { complaints }\end{array}$ & \begin{tabular}{|l|} 
Intro-jection I \\
Retro-flexion
\end{tabular} & Sociabi-lity & High intelli-gence & Diplo-macy & Con-formism & (I) in obstruct-tion situation \\
\hline \multirow{3}{*}{ 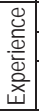 } & Pearson Correla-tion & $350^{* *}$ &, $231^{* *}$ & $247^{\text {*t+ }}$ & $267^{* *}$ &, $248^{* *}$ &,$- 227^{\text {* }}$ &,$- 256^{* *}$ \\
\hline & Signifi-cance (2-sided) &, 000 & 004 & 002 & 001 & 002 & ,004 & 001 \\
\hline & $\mathrm{N}$ & 156 & 156 & 156 & 156 & 156 & 156 & 156 \\
\hline
\end{tabular}

Analyzing the correlation of psychological practice duration and personal features of the testees, their strategy of behavior in frustration situations suggests that the characteristics typical for the second group (such as "Restraint", "Conformism") may cause a trend towards the use of psychological defense "Deflexion", which is characterized by avoidance of contact, closed borders, isolation, focusing on Ego. The psychologists of second group have crossed the line of choosing "Is this a job I want to do, Is it is my field of activity", they have already chosen their path, but, nevertheless, the process of becoming a professional still continues. And that's why the research subjects are currently at risk group of emotional burnout and professional deformation. Perhaps, increase in impunitive reactions in the second group was transformed into the feature "Diplomacy" in the third group.

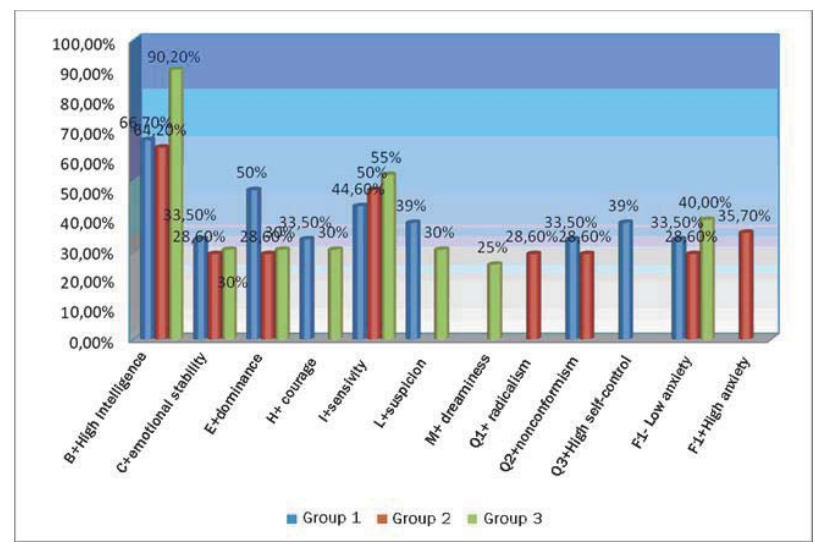

Diagram 2. Extent of personal features of the testees from the three groups according to the Cattell's test

Let us look at the results, obtained using the Rosenzweig methods of frustration reactions study. The prevalent answers of the research subjects in the first group were impunitive (50\%) and extrapunitive (44.5\%) reactions with focusing on self-defense (44.5\%) satisfaction of needs (44.5\%). Thus, the situations of frustration are treated by the first group of the testees as desperately important, the internal and external causes of frustration are underlined. In their answers a great role is devoted to satisfaction of their needs and shifting responsibilities in resolving the situation to other persons, blaming somebody, denial or admission of personal guilt. Also the GCR- coefficient of group conformity or extent of individual adaptation of subject to its social environment was calculated. Quantitative average GCR value in the first group comprised 44.4 scores. This is an average level. This indicator shows a good adaptation to the social environment, mutual understanding and a constructive behavior in most cases (Maslach, 1976).

The results of Pearson correlation analysis showed that with increase of GCR indicator in the first group that is with 
increase of adaptation to social environment, the "Rheumatic complaints" "Trustfulness", "Straightforwardness" and "Calmness" (Cattell's test) increase, the reactions of NP-focusing on satisfaction of needs reduce, extrapunitive reactions in the obstruction situation reduce. The increase in group conformity involves the development of a trusting relationship with the group, and as a consequence the trustfulness to the participants of communication increases, openness in judgments and statements, strive to solve problems independently disappears, the reactions of judging and blaming the participants of communication disappear, and there is a risk of increasing of "rheumatic complaints.

Let us look at the results, obtained using the Rosenzweig methods of frustration reactions study in the second group. The prevalent answers of the research subjects in the second group were impunitive (75\%) reactions with focusing on self-defense (71.4\%). Frustrating situation is treated by the testees as not important, as the absence of anyone's fault or as something that may be solved itself, one has only to wait and think, the defense of themselves, their "Ego" plays the main role in their answers, the testees criticize anyone or admit their guilt . Quantitative average value of GCR coefficient in the second group was 34 scores - this is the average level of adaptation to the social environment. $71.4 \%$ of the testees in the second group have average GCR coefficient (see Diagram). This indicator shows an average, close to low, level of adaptation to the social environment. This level of group conformity serves as an indicator of low adaptation to social environment and proneness to conflict, resulting in reducing frustration tolerance, which in turn becomes a cause of view of world as less safe. This leads to increasing of the proportion of ego-defensive reactions in the form of verbal aggression and criticizing a partner as situations demanded.

The Pearson correlation analysis of the GCR indicator in the second group revealed a positive relationship $(r=$ $0.350 ; p=0.009)$ with the scale of "Rheumatic complaints" and negative relationship $(r=-0.433 ; p=0.001)$ with intropunitive reactions in the obstruction situation. With the increase of the level of group conformity among the participants of the second group the rheumatic complaints increase and auto-directed reactions in a situation of frustration reduce.

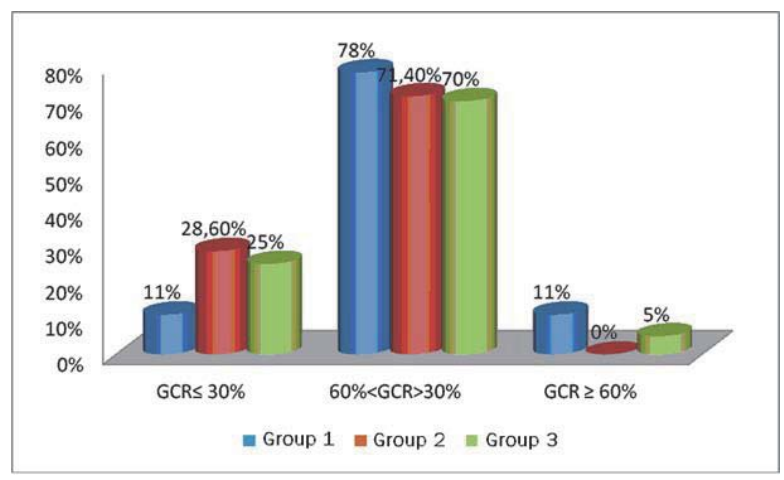

Diagram 3. Representation of GCR indicator (adaptation to the social environment), of the testees of three groups

Next, let us look at the results, obtained using the Rosenzweig methods of frustration reactions study in the third group. The prevalent answers of the testees in the third group were impunitive (47,5\%) and extrapunitive (40\%) reactions with focusing on self-defense (53\%) and satisfaction of needs (36\%). Frustrating situation is treated by the testees as not important, as the absence of anyone's fault or as something that may be solved itself, one has only to wait and think, and also they have the reaction referred to the live or abiotic environment in form of accentuation of severity of frustrating situation. The defense of themselves, their "Ego" plays the main role in their answers, the testees criticize anyone or admit their guilt . Quantitative average value of GCR coefficient in the second group was 35,9 scores - this is the average level of adaptation to the social environment. $70 \%$ of the testees in the third group have average GCR indicator (see Diagram). This indicator shows an average level of adaptation to the social environment. This level of group conformity serves as an indicator of low adaptation to social environment and proneness to conflict, resulting in reducing frustration tolerance, which in turn becomes a cause of view of world as less safe. This leads to increasing of the proportion of egodefensive reactions in the form of verbal aggression and criticizing a partner as situations demanded.

The analysis revealed in the third group a weak positive correlation of low statistical significance of GCR indicator and "Rheumatic complaints" although the percentage of participants with prominent "Rheumatic complaints" has grown in comparison with other groups. 


\section{Conclusion and Findings}

Such qualities, as wariness towards people, propensity to jealousy, desire to lay responsibility for errors on others, and sourness are typical of the research subjects at the initial stage of their psychological practice. These qualities can promote reduced use of the "Introjection / Retroflexion" psychological defense, a possible increase in somatic complaints, and as a result, the development of professional deformation.

The specific character of professional activity of a psychologist is an objective factor of professional deformation occurrence. At the initial stage of the professional activity the "Pressure of somatic complaints" is formed by more frequent "Exhaustion" and "Rheumatic complaints". With a length of 2 to 5 years work experience, the higher incidence of "Pressure of somatic complaints" is due to more frequent "Rheumatic complaints" and "Gastric complaints". For over 5year work experience, the "Pressure of somatic complaints" is formed due to "Exhaustion" and "Heart complaints", and it is by the pronounced "Heart complaints" that this group differs from the others.

The use of "Introjection / Retroflexion" psychological defense by psychologists having an over 5-year work experience helps them to reduce reactions aimed at the environment, the trend towards self-justification and defense of Ego, and to increase the trends towards searching for constructive solutions of difficulties and conflicts arising, as well as to reduce the trend to blame the others in case of obstacles. However, this, in its turn, can lead to the formation of "Heart complaints".

The use of the "Projection" defense mechanism may promote the increase of somatic "Exhaustion" and "Pressure of somatic complaints".

Higher group conformity implies the development of trusting relationships with the group, and as a consequence, trustfulness to the participants of communication increases, and so does openness in judgments and statements, while the desire to solve problems independently disappears, and the reactions of judging and blaming the participants of communication go away too. However, there is a risk of more frequent "Rheumatic complaints".

Using the "Deflexion" defense promotes the formation of "Gastric complaints". Metaphorically speaking, "closedness" to contacts with other people, unwillingness to share feelings and emotions, keeping one's reactions to oneself may lead to failure to bear the clients while working on their problems, i.e. to a kind of "indigestion", and, as a result, the development of "Gastric complaints". Thus, the second group (having 2 to 5 -year professional activity) is a critical one and is in the "risk group" as for a practicing psychologist's professional deformation development.

\section{References}

Boiko, V.V. (1996). Energiya emotsiy v obschenii: vzglyad na sebya i na drugih. [Energy of emotion in communication: a look at oneself and at others]. Moskva.

Greenberger, R.S. (1981). Job hazard - how "burnout" affects corporate managers and their performances. The Wall Street Journal.

Makarova, G.A. Sindrom emotsionalnogo vygoraniya [Emotional burnout syndrome]. [Electronic resource]. URL: http://www.myword.ru

Maslach, C. (1978). Job burnout - how people cope. Public Welfare. Spring.

Maslach, C. (1976). Burned-out. Human Behavior, № 5.

Miroshnichenko, S.S. (2014) Deformation of the semantic sphere of adolescents who have communicative disorders. World Applied Sciences Journal.

Orel, V.E. (2001). Fenomen "vygoraniya" v zarubezhnoi psihoogii: empiricheskiye issledovaniya [Phenomenon of "burnout" in foreign psychology: empirical studies.// Psychological Journal, V. 15, No. 1, 89-99.

Pines, A., Maslach, C. (1978). Characteristics of staff burnout in mental health settings // Hosp. Community Psychiat., № 29.

Samoukina, N.V. Sindrom professionalnogo vygoraniya. [Professional burnout syndrome] [Electronic resource]. URL: http://www.elitarium.ru/2005/01/12/sindrom_professionalnogo_vygoranija.html

Sidorov, P.I. Sindrom professionalnogo vygoraniya. Konspekt vracha [Professional burnout syndrome. A doctor's notes] [Electronic resource]. URL: http://health.mpei.ac.ru/sindrom.htm

Vodopianova, N.E., Starchenkova, E.S. (2009). Sindrom vygoraniya: diagnostika i profilaktika [Burnout syndrome: diagnosis and prevention]. SPb.: Piter. 